\title{
Perfil radial e uniformidade de precipitação do aspersor NaanD anJain 427, em função da regulagem do defletor
}

\author{
Paulo E. S. Martins ${ }^{1}$, José R. Zanini², Elcides R. da Silva ${ }^{1} \&$ Marco A. F. Lemos Filho ${ }^{3}$ \\ RESU MO \\ Neste trabalho o objetivo foi avaliar o perfil radial e a uniformidade de distribuição de água do aspersor \\ fabricado pela empresa NaanDanjain, modelo $4271 / 2 " \mathrm{M}$ e bocal de $2,8 \mathrm{~mm}$ de diâmetro interno, \\ operando com pressões de 150, 200, 300 e $400 \mathrm{kPa}$ em cinco posições do defletor de ajuste $(0,20,50$, \\ 80 e $100 \%$ ). Para a determinação dos parâmetros avaliados utilizou-se o método da malha e, com 0 \\ auxílio do aplicativo computacional CATCH 3D, calculou-se a sobreposição das lâminas de água com \\ dez espaçamentos. Os resultados obtidos demonstram que o defletor de ajuste influencia o raio de \\ molhamento e o perfil de distribuição enquanto a uniformidade de aplicação de água se mostrou como \\ mecanismo importante, visto que permite comportamento diferenciado para o aspersor, garantindo \\ ampla faixa de utilização do equipamento.
}

Palavras-chave: uniformidade da irrigação, perfil de distribuição de água, desempenho hidráulico

\section{Radial profile and precipitation uniformity of sprinkler NaanDanJain 427, depending on the setting of the deflector}

\begin{abstract}
The study aimed to evaluate the radial profile and the uniformity of water distribution of sprinkler manufactured by the company NaanDanJain, model $4271 / 2 " \mathrm{M}$ and nozzle with $2.8 \mathrm{~mm}$ of internal diameter, operating at pressures of $150,200,300$ and $400 \mathrm{kPa}$ and five positions of the deflector $(0,20$, $50,80$ and $100 \%)$. For the determination of the parameters evaluated, the grid method was used and with the help of computer application CATCH 3D, overlapping layers of water depths was calculated with ten spacing. The results show that the deflector adjustment influences the radius of wetness and the distribution profile while the uniformity of water application showed as an important mechanism, since it permits different behavior for the sprinkler, ensuring wide track of utilization of the equipment.
\end{abstract}

Key words: irrigation uniformity, water distribution profile, hydraulic performance

\footnotetext{
${ }^{1}$ Doutorando do Programa de Pós-graduação em Ciência do Solo da Universidade Estadual Paulista "Júlio de Mesquita Filho" - Câmpus de Jaboticabal. Departamento de Engenharia Rural. Via de Acesso Paulo D onato Castellane, s/n. CEP: 14884-900. Jaboticabal - SP. Fone: (016) 3209-2600. E-mail: paulo_xx_martins@hotmail.com; rodagro@hotmail.com

2 Universidade Estadual Paulista "Júlio de Mesquita Filho" - Câmpus de Jaboticabal. E-mail: jrzanini@fcav.unesp.br

3 U niversidade Estadual Paulista "Júlio de Mesquita Filho" - Câmpus de Jaboticabal. E-mail: maflf@hotmail.com
} 


\section{INTRODUÇÃO}

A irrigação é muito importante para o desenvolvimento da agricultura, pois permite a estabilidade da produção em períodos de déficit hídrico. Nesse contexto, a agricultura irrigada tem alcançado expressividade no setor produtivo mundial, com área irrigada em torno de 278 milhões de ha, o que ampliou a área cultivada sob irrigação, para $18 \%$ do total, responsável por $44 \%$ da produção mundial de alimentos (Christofidis, 2008); entretanto, a crescente utilização da água para fins de irrigação tem despertado a preocupação da opinião pública e das lideranças políticas, em razão dos impactos causados ao ambiente pela má utilização das águas pelos irrigantes. Segundo Andrade et al. (2004), a agricultura irrigada tem afetado o meio ambiente com impactos que podem conduzir à degradação do solo e das águas, com perda de produtividade.

Coelhoet al. (2005) citaram que a simples melhoria de $1 \%$ na eficiência do uso da água de irrigação nos países em desenvolvimento de clima árido e semiárido, significaria economia de cerca de 200 mil L de água por agricultor ha $^{-1}$ ano $^{-1}$.

A desuniformidade de distribuição de água dos sistemas acarretando excesso de aplicação de água em parte da área irrigada pode acarretar, também, outros problemas ambientais, como a lixiviação de fertilizantes e contaminação de águas subterrâneas, devido à percolação profunda (Capra \& Scicolone, 2004; Oliveira et al., 2009; Paulino et al., 2009).

No caso da aspersão convencional os cuidados devem ser maiores quando se trata das perdas durante a aplicação de água, as quais são mais significativas e dependentes do equipamento em uso. Christiansen (1942) e Hart et al. (1979) citam que o desempenho de um sistema de irrigação por aspersão pode ser analisado utilizando-se a uniformidade de distribuição de água e a eficiência de aplicação, de vez que expressam a qualidade da irrigação e se tornam decisivas no planejamento e na operação desses sistemas.

O Coeficiente de Uniformidade de Christiansen (CUC) é considerado, por muitos autores, o parâmetro principal para calcular a uniformidade de irrigação, sendo usado para medir a variabilidade espacial da lâmina de água aplicada pelo sistema de irrigação. Um sistema com CUC de $80 \%$ significa que aproximadamente $80 \%$ da área receberão lâmina maior ou igual à lâmina média de aplicação; já o Coeficiente de Uniformidade de Distribuição (CUD) relaciona as menores lâminas aplicadas no quartil da área total (quartil que recebe menos água) com a lâmina média aplicada. Caso toda a área receba no mínimo a lâmina real necessária, baixo valor de CUD indica perda excessiva por percolação profunda (Rezende et al., 1998).

Altos valores de uniformidade de aplicação de água são fundamentais para a obtenção da maior produtividade de uma cultura com menor consumo de água, energia e fertilizantes (Álvarez et al., 2004). De acordo com Louie \& Selker (2000), no cenário atual, em que a proteção ambiental e a conservação dos recursos hídricos são enfatizadas e os custos de energia e água estão em ascensão, é crescente a preocupação com a uniformidade de aplicação de água pelos fabricantes de aspersores, projetistas e usuários de sistemas de irrigação. Entretanto e segundo Prado \& Colombo (2009), a existência de inúmeras possibilidades de combinação de pressão de serviço e diâmetro de bocais, leva os fabricantes a não disponibilizarem ou a não apresentarem, de maneira adequada, as características técnicas de distribuição de água de seus aspersores. Como alternativa para a caracterização de aspersores com economia de recursos materiais e financeiros, além de ganho de tempo, Borges Júnior et al. (2008) citaram o uso de modelos computacionais para a simulação da uniformidade de distribuição de água em diversas condições de espaçamento.

O defletor existente nos aspersores contribui para o fracionamento do jato que sai através do bocal, com o intuito de melhorar a uniformidade de distribuição de água. $\mathrm{O}$ conhecimento de sua atuação permite, ao projetista e ao usuário do sistema de irrigação, a utilização do aspersor em maior amplitude de situações, tais como: necessidade de alterações do raio de molhamento, em função da fase da cultura, do formato da parcela irrigada e do espaçamento entre aspersores.

Diante do exposto e com o atual apelo ambiental relacionado ao consumo de água, o presente estudo teve como objetivo avaliar o perfil radial e a uniformidade de distribuição de água do aspersor fabricado pela empresa NaanDanJain, modelo 427 1/2" Me bocal de 2,8 mm de diâmetro interno, operando com quatro pressões em cinco posições do defletor de ajuste.

\section{Material e MÉTOdos}

Este trabalho foi conduzido em área situada no Departamento de Engenharia Rural da Faculdade de Ciências Agrárias e Veterinárias, Universidade Estadual Paulista, Campus de Jaboticabal e desenvolvido com aspersor de plástico, de impacto, rotativo, com bocal de diâmetro interno de $2,8 \mathrm{~mm}$, fabricado pela empresa NaanDanJain, modelo 427 1/2" M utilizado, em geral, para irrigação em parques, jardins e culturas.

$O$ aspersor possui defletor de ajuste que possibilita modificar o perfil radial de distribuição de água em função de onze posições, variando o comprimento do defletor (Figura 1) a cada $1 \mathrm{~mm}$ a partir da posição inicial $0 \mathrm{~mm}(0 \%)$ até a posição final de $10 \mathrm{~mm}$ (100\%), de forma a aumentar, em cada posição, o grau de

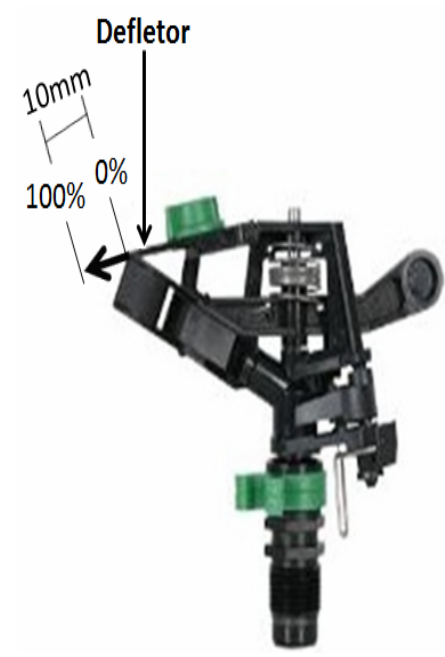

Figura 1. Aspersor 427 1/2"M com expansão do defletor de ajuste de 0 a $100 \%$ de seu comprimento 
interferência do defletor sobre o jato. Deste modo, foram avaliadas cinco posições $(0 ; 20 ; 50 ; 80$; e $100 \%)$ com três repetições, representadas por três aspersores distintos.

De acordo com o catálogo do fabricante (Tabela 1), para o bocal de 2,8 $\mathrm{mm}$ de diâmetro interno as vazões variam de 0,38 a $0,63 \mathrm{~m}^{3} \mathrm{~h}^{-1}$ para pressões de 150 a $400 \mathrm{kPa}$ e os diâmetros de molhamento se situam entre 21 e $24 \mathrm{~m}$, respectivamente. Os testes foram realizados de acordo com as pressões sugeridas pelo fabricante $(150,200,300$ e $400 \mathrm{kPa})$.

Tabela 1. Vazões (Q) ) e diâmetros de molhamento (D) do aspersor 427 1/2"M em função da pressão (P) para o bocal de $2,8 \mathrm{~mm}$ de diâmetro interno

\begin{tabular}{cccc}
\hline \multicolumn{4}{c}{ Tabela de desempenho } \\
\hline $\begin{array}{c}\text { Diâmetro do bocal } \\
(\mathbf{m m})\end{array}$ & $\begin{array}{c}\mathbf{P} \\
(\mathbf{k P a})(\mathbf{b a r})\end{array}$ & $\begin{array}{c}\mathbf{Q} \\
\left(\mathbf{m}^{\mathbf{3}} \mathbf{h}^{-\mathbf{1}}\right)\end{array}$ & $\begin{array}{c}\mathbf{D} \\
(\mathbf{m})\end{array}$ \\
& $150(1,5)$ & 0,380 & 21,0 \\
2,8 & $200(2,0)$ & 0,450 & 22,0 \\
& $300(3,0)$ & 0,550 & 23,0 \\
& $400(4,0)$ & 0,630 & 24,0 \\
\hline
\end{tabular}

Fonte: NaanDanj ain, 2010

Para a determinação dos parâmetros avaliados utilizou-se metodologia descrita pela ABNT (1999), chamada método da malha, fazendo-se um espalhamento dos coletores igualmente espaçados, formando uma malha ao redor do aspersor. Os coletores foram espaçados $2,0 \mathrm{~m}$ a partir do aspersor, formando quatro quadrantes em uma superfície pavimentada. Foram dispostas, também, duas linhas radiais de coletores espaçados $1,0 \mathrm{~m}$ a partir do aspersor, visando à obtenção do perfil radial de distribuição de água do aspersor (Figura 2). Para se obter valores de precipitação correspondentes a aproximadamente 0 $\mathrm{m}$ da distância do aspersor, foram colocados também três coletores dispostos triangularmente a $0,05 \mathrm{~m}$ do tubo de elevação do aspersor.

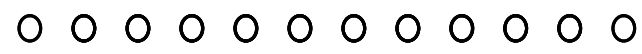

000000000000

000000000000

000000000000

000000000000

000000000000 $00000000000 * 00000000000$ 000000000000

000000000000

000000000000

000000000000

000000000000

0000000000000

Figura 2. Esquema de distribuição dos coletores
Os coletores utilizados foram da marca Fabrimar, que possuem forma geométrica tronco-cônica, com diâmetro $80 \mathrm{~mm}$ na parte superior e altura de $102 \mathrm{~mm}$. O volume armazenado dentro de cada coletor foi medido com provetas graduadas de 10 e $25 \mathrm{~mL}$.

$\mathrm{O}$ aspersor foi instalado na altura de $0,7 \mathrm{~m}$ em relação à superfície e, para a determinação da vazão, fez-se o teste do volume de água coletado em função do tempo e da pressão de trabalho. A pressão de operação para cada teste foi controlada utilizando-se dois manômetros de Bourdon, acoplados um na base do aspersor e o outro na saída da bomba, previamente calibrados em laboratório com manômetro de coluna de mercúrio. A pressão foi ajustada com registros de gaveta e de agulha, instalados na saída da bomba e na haste de subida do aspersor, respectivamente.

As repetições das avaliações foram realizadas com três aspersores para o que se realizaram, inicialmente, testes com os aspersores determinando-se as vazões nas pressões de teste, possibilitando calcular o coeficiente de variação de fabricação (CVF) (Eq. 1) dos mesmos e permitindo comprovar a representatividade dos aspersores como repetições, através de baixo CVF.

$$
\mathrm{CVf}=100 \frac{\sqrt{\left(\mathrm{q}_{1}{ }^{2}+\mathrm{q}_{2}{ }^{2}+\ldots \mathrm{q}_{\mathrm{n}}{ }^{2}-\mathrm{nq}_{\mathrm{m}}{ }^{2}\right) \div(\mathrm{n}-1)}}{\mathrm{q}_{\mathrm{m}}}
$$

em que:

CVf- coeficiente de variação de fabricação, \%

$\mathrm{q}_{1}, \mathrm{q}_{2, \ldots} \mathrm{q}_{\mathrm{n}}$ - vazão de cada emissor, $\mathrm{L} \mathrm{h}^{-1}$

$\mathrm{q}_{\mathrm{m}}$ - vazão média dos emissores, $\mathrm{L} \mathrm{h}^{-1}$

n - número de emissores do lote de amostragem

Obtiveram-se os dados de direção e velocidade do vento, no local da área de testes. A velocidade do vento foi medida com um anemômetro no decorrer dos testes, instalado a 2,0 m da superfície pavimentada. Os ensaios para determinação da característica técnica do aspersor (vazão e perfil radial de distribuição de água) foram realizados sob condições de vento inferiores a 2,0 $\mathrm{m} \mathrm{s}^{-1}$, conforme especificação da ABNT (1999). Para medir a evaporação durante o teste foram colocados, ao lado da malha de coletores, cinco coletores iguais aos usados como pluviômetros, com 1, 3, 5, 7 e 9 mm de lâmina d'água para a estimativa da média de evaporação. O tempo de cada teste foi de 60 minutos, tempo após o qual os volumes coletados foram anotados em planilhas apropriadas. Para a representação do volume relativo à posição para cada coletor foi calculada a média do volume dos coletores de cada aspersor.

A metodologia para avaliação da uniformidade de irrigação foi baseada nos índices CUC (Christiansen, 1942) e CUD (Criddle et al., 1956). Foram analisados, também, os efeitos das pressões de serviço e dos espaçamentos dos aspersores sobre a uniformidade de distribuição de água.

A interpretação dos valores de CUC e CUD baseou-se na proposição de Mantovani (2001), apresentada na Tabela 2.

O aplicativo computacional Sprinkler Catch CanOverlapProgram - CATCH 3D, versão 4.45 (Allen, 1992), 
Tabela 2. Classificação dos valores de CUC e CUD para sistemas de aspersão

\begin{tabular}{lcc}
\hline Classificação & CUC & CUD \\
Excelente & $>90$ & (\%) \\
Bom & $80-90$ & $>84$ \\
Razoável & $70-80$ & $68-84$ \\
Ruim & $60-70$ & $52-68$ \\
Inaceitável & $<60$ & $36-52$ \\
\hline
\end{tabular}

Fonte: Adaptado de Mantovani (2001)

foi utilizado para calcular a sobreposição com diferentes espaçamentos. Os resultados relativos à sobreposição e determinação da uniformidade de distribuição de água pelo aspersor foram baseados nos dados de precipitação, sendo simulados espaçamentos $(6 \times 6,6 \times 8,6 \times 10,6 \times 12,8 \times 8,8 \times 10$, $8 \times 12,10 \times 10,10 \times 12$ e $12 \times 12$ m) entre aspersores e entre linhas laterais, respectivamente.

Ajustaram-se, com base nas vazões médias para cada pressão, as equações características, conforme Keller \& Karmeli (1974) (Eq. 2):

$$
\mathrm{Q}=\mathrm{KH}^{\mathrm{n}}
$$

em que:

$\mathrm{Q}$ - vazão, $\mathrm{m}^{3} \mathrm{~h}^{-1}$

$\mathrm{H}$ - pressão, $\mathrm{kPa}$

K e n - parâmetros de ajuste.

\section{RESULTADOS E DISCUSSÃO}

\section{Coeficiente de variação de fabricação (CVf)}

Os efeitos dos fatores relacionados à fabricação para o aspersor Naan Dan Jain 427 de bocal com 2,8 mm de diâmetro e o defletor na posição de $0 \%$, nas pressões estudadas, estão apresentados na Tabela 3.

Tabela 3. D ados do coeficiente de variação de fabricação (CVf) do aspersor NaanDanJain 427 de bocal com diâmetro interno de $2,8 \mathrm{~mm}$

\begin{tabular}{ccc}
\hline $\begin{array}{c}\text { Pressão } \\
(\mathbf{k P a})\end{array}$ & $\begin{array}{c}\text { Vazão } \\
\left(\mathbf{m}^{\mathbf{3}} \mathbf{h}^{-1}\right)\end{array}$ & $\begin{array}{c}\text { CVf } \\
(\%)\end{array}$ \\
150 & 0,44 & 1,69 \\
200 & 0,52 & 1,83 \\
300 & 0,64 & 2,10 \\
400 & 0,72 & 2,43 \\
\hline
\end{tabular}

Verifica-se que o aspersor NaanDanJain 427 apresentou baixo CVf, variando entre 1,69 e 2,43\%, mostrando pequena variação no fluxo dos emissores devido ao processo de fabricação. A importância da obtenção de CVf de baixo valor é justificada por Keller \& Karmeli (1974), ao afirmarem que a uniformidade de distribuição de água é dependente também da precisão com que os aspersores são fabricados.

A avaliação do efeito da pressão no CVf mostrou efeito linear da pressão sobre o CVf. Constatou-se aumento do CVf à medida em que a pressão aumenta (Figura 3). Resultados similares já haviam sido encontrados por Holanda Filho et al. (2001) com o microaspersor Rondo $\left(50 \mathrm{~L} \mathrm{~h}^{-1}\right)$ da empresa Plastro.

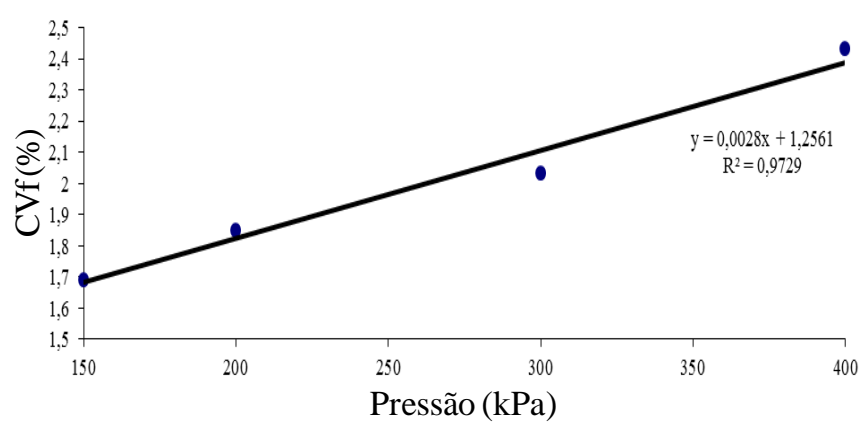

Figura 3. Valores médios dos Coeficientes de Variação de Fabricação (CVf) para o aspersor $\mathrm{N}$ aanD anjain 427 com bocal de 2,8 $\mathrm{mm}$ de diâmetro interno

\section{Equação característica vazão-pressão}

A partir dos dados de vazão medidos em cada pressão através de análise de regressão foi determinado a equação característica do aspersor, sendo: $\mathrm{Q}=0,0357 \times \mathrm{H}^{0,5036}$ e o coeficiente de determinação $\left(\mathrm{R}^{2}\right)$ igual a 0,9957 (Figura 4), demonstrando bom ajuste dos dados observados.

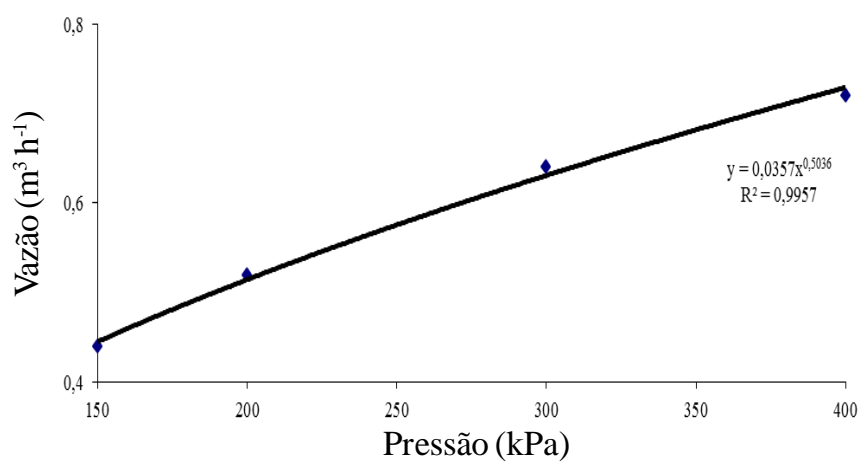

Figura 4. Curva característica vazão-pressão para o aspersor $\mathrm{N}$ aanDanjain 427 com bocal de $2,8 \mathrm{~mm}$ de diâmetro interno

Na Tabela 4 se apresentam os valores médios da vazão em função da pressão, obtidos durante os ensaios, junto aos erros estimados entre as vazões informadas pelo fabricante e as vazões médias obtidas durante os ensaios. Foram encontrados erros superiores a 5\%, limite aceitável pela ABNT (1999).

Tabela 4. Comparação entre vazões encontradas durante os ensaios e vazões fornecidas pelo fabricante

\begin{tabular}{cccc}
\hline $\begin{array}{c}\text { Pressão } \\
\mathbf{( k P a )}\end{array}$ & $\begin{array}{c}\text { Vazão obtida } \\
\left(\mathbf{m}^{\mathbf{3}} \mathbf{h}^{-\mathbf{1}}\right)\end{array}$ & $\begin{array}{c}\text { Vazão fornecida } \\
\left(\mathbf{m}^{\mathbf{3}} \mathbf{h}^{-\mathbf{1}}\right)\end{array}$ & $\begin{array}{c}\text { Erro } \\
(\%)\end{array}$ \\
150 & 0,44 & 0,38 & 15,78 \\
200 & 0,52 & 0,45 & 15,55 \\
300 & 0,64 & 0,55 & 16,36 \\
400 & 0,72 & 0,63 & 14,28 \\
\hline
\end{tabular}

\section{Uniformidade de distribuição de água}

Nas Tabelas 5, 6, 7 e 8 tem-se os valores médios de CUC, CUD e raio de molhamento para os espaçamentos adotados $(6$ x 6,6 × 8,6 × 10,6 x 12, 8 x 8, 8 x 10, 8 x 12, 10 x 10, 10 x 12 e 12 x 
Tabela 5. Valores médios obtidos de CU C (\%), CUD (\%) e raio de molhamento (Raio) para a pressão de $150 \mathrm{kPa}$, em função do espaçamento entre aspersores, calculados pelo aplicativo CATCH-3D

\begin{tabular}{|c|c|c|c|c|c|c|c|c|c|c|c|c|}
\hline \multirow{2}{*}{$\begin{array}{c}\text { Defletor } \\
(\%)\end{array}$} & \multirow{2}{*}{ CUC e CUD } & \multicolumn{10}{|c|}{ Espaçamento entre aspersores ( $\mathrm{m} \times \mathrm{m}$ ) } & \multirow{2}{*}{$\begin{array}{l}\text { Raio } \\
\text { (m) }\end{array}$} \\
\hline & & $6 \times 6$ & $6 \times 8$ & $6 \times 10$ & $6 \times 12$ & $8 \times 8$ & $8 \times 10$ & $8 \times 12$ & $10 \times 10$ & $10 \times 12$ & $12 \times 12$ & \\
\hline 0 & $\begin{array}{l}\text { CUC } \\
\text { CUD }\end{array}$ & $\begin{array}{l}89,8 \\
88,5\end{array}$ & $\begin{array}{l}79,7 \\
70,7\end{array}$ & $\begin{array}{l}87,7 \\
85,6\end{array}$ & $\begin{array}{l}84,7 \\
75,5\end{array}$ & $\begin{array}{l}74,2 \\
49,6\end{array}$ & $\begin{array}{l}79,9 \\
67,8\end{array}$ & $\begin{array}{l}80,2 \\
65,7\end{array}$ & $\begin{array}{l}85,0 \\
77,9\end{array}$ & $\begin{array}{l}75,3 \\
58,2\end{array}$ & $\begin{array}{l}72,1 \\
59,1\end{array}$ & 11 \\
\hline 20 & $\begin{array}{l}\text { CUC } \\
\text { CUD }\end{array}$ & $\begin{array}{l}88,6 \\
86,0\end{array}$ & $\begin{array}{l}78,6 \\
72,9\end{array}$ & $\begin{array}{l}86,5 \\
79,2\end{array}$ & $\begin{array}{l}83,5 \\
74,4\end{array}$ & $\begin{array}{l}72,6 \\
50,4\end{array}$ & $\begin{array}{l}79,1 \\
66,8\end{array}$ & $\begin{array}{l}80,2 \\
67,6\end{array}$ & $\begin{array}{l}80,0 \\
70,6\end{array}$ & $\begin{array}{l}71,5 \\
50,9\end{array}$ & $\begin{array}{l}67,5 \\
46,7\end{array}$ & 11 \\
\hline 50 & $\begin{array}{l}\text { CUC } \\
\text { CUD }\end{array}$ & $\begin{array}{l}90,6 \\
88,7\end{array}$ & $\begin{array}{l}78,6 \\
73,1\end{array}$ & $\begin{array}{l}89,2 \\
81,9\end{array}$ & $\begin{array}{l}82,8 \\
70,4\end{array}$ & $\begin{array}{l}72,7 \\
49,2\end{array}$ & $\begin{array}{l}78,8 \\
65,9\end{array}$ & $\begin{array}{l}78,2 \\
64,0\end{array}$ & $\begin{array}{l}82,3 \\
73,7\end{array}$ & $\begin{array}{l}72,0 \\
55,7\end{array}$ & $\begin{array}{l}70,8 \\
55,4\end{array}$ & 11 \\
\hline 80 & $\begin{array}{l}\text { CUC } \\
\text { CUD }\end{array}$ & $\begin{array}{l}94,5 \\
90,3\end{array}$ & $\begin{array}{l}79,2 \\
73,7\end{array}$ & $\begin{array}{l}57,8 \\
20,5\end{array}$ & $\begin{array}{r}39,7 \\
5,8\end{array}$ & $\begin{array}{l}74,0 \\
51,4\end{array}$ & $\begin{array}{l}53,9 \\
26,7\end{array}$ & $\begin{array}{r}33,8 \\
5,2\end{array}$ & $\begin{array}{r}37,4 \\
8,1\end{array}$ & $\begin{array}{r}20,0 \\
0,0\end{array}$ & $\begin{array}{l}4,4 \\
0,0\end{array}$ & 6 \\
\hline 100 & $\begin{array}{l}\text { CUC } \\
\text { CUD }\end{array}$ & $\begin{array}{l}91,1 \\
83,4\end{array}$ & $\begin{array}{l}66,9 \\
57,1\end{array}$ & $\begin{array}{r}46,9 \\
7,8\end{array}$ & $\begin{array}{r}30,7 \\
2,2\end{array}$ & $\begin{array}{l}63,2 \\
33,7\end{array}$ & $\begin{array}{l}40,0 \\
14,0\end{array}$ & $\begin{array}{r}20,0 \\
1,9\end{array}$ & $\begin{array}{r}18,4 \\
3,0\end{array}$ & $\begin{array}{l}2,4 \\
0,0\end{array}$ & $\begin{array}{l}0,0 \\
0,0\end{array}$ & 5 \\
\hline
\end{tabular}

Legenda: Conjuntos de dados (CUC e CUD) com hachuras representam valores combinados e considerados excelentes por Mantovani (2001)

Tabela 6. Valores médios obtidos de CUC (\%), CUD (\%) e raio de molhamento (Raio) para a pressão de $200 \mathrm{kPa}$ em função do espaçamento entre aspersores, calculados pelo aplicativo CATCH-3D

\begin{tabular}{|c|c|c|c|c|c|c|c|c|c|c|c|c|}
\hline \multirow{2}{*}{$\begin{array}{c}\text { Defletor } \\
(\%)\end{array}$} & \multirow{2}{*}{ CUC e CUD } & \multicolumn{10}{|c|}{ Espaçamento entre aspersores ( $\mathrm{m} \times \mathrm{m})$} & \multirow{2}{*}{$\begin{array}{l}\text { Raio } \\
\text { (m) }\end{array}$} \\
\hline & & $6 \times 6$ & $6 \times 8$ & $6 \times 10$ & $6 \times 12$ & $8 \times 8$ & $8 \times 10$ & $8 \times 12$ & $10 \times 10$ & $10 \times 12$ & $12 \times 12$ & \\
\hline 0 & $\begin{array}{l}\text { CUC } \\
\text { CUD }\end{array}$ & $\begin{array}{l}93,1 \\
92,9\end{array}$ & $\begin{array}{l}82,4 \\
71,0\end{array}$ & $\begin{array}{l}86,4 \\
80,9\end{array}$ & $\begin{array}{l}91,2 \\
86,3\end{array}$ & $\begin{array}{l}79,5 \\
62,0\end{array}$ & $\begin{array}{l}80,2 \\
65,5\end{array}$ & $\begin{array}{l}84,0 \\
73,3\end{array}$ & $\begin{array}{l}83,0 \\
77,0\end{array}$ & $\begin{array}{l}80,1 \\
65,2\end{array}$ & $\begin{array}{l}76,6 \\
62,4\end{array}$ & 12 \\
\hline 20 & $\begin{array}{l}\text { CUC } \\
\text { CUD }\end{array}$ & $\begin{array}{l}93,1 \\
92,9\end{array}$ & $\begin{array}{l}84,5 \\
76,3\end{array}$ & $\begin{array}{l}91,1 \\
85,0\end{array}$ & $\begin{array}{l}90,2 \\
80,6\end{array}$ & $\begin{array}{l}81,2 \\
63,2\end{array}$ & $\begin{array}{l}84,9 \\
72,4\end{array}$ & $\begin{array}{l}83,8 \\
73,9\end{array}$ & $\begin{array}{l}89,0 \\
85,0\end{array}$ & $\begin{array}{l}81,0 \\
63,3\end{array}$ & $\begin{array}{l}77,6 \\
61,5\end{array}$ & 11 \\
\hline 50 & $\begin{array}{l}\text { CUC } \\
\text { CUD }\end{array}$ & $\begin{array}{l}92,6 \\
90,1\end{array}$ & $\begin{array}{l}80,7 \\
73,8\end{array}$ & $\begin{array}{l}90,5 \\
85,8\end{array}$ & $\begin{array}{l}86,1 \\
80,7\end{array}$ & $\begin{array}{l}76,2 \\
52,5\end{array}$ & $\begin{array}{l}79,0 \\
69,0\end{array}$ & $\begin{array}{l}79,2 \\
65,7\end{array}$ & $\begin{array}{l}85,5 \\
81,6\end{array}$ & $\begin{array}{l}76,4 \\
61,6\end{array}$ & $\begin{array}{l}75,2 \\
62,0\end{array}$ & 11 \\
\hline 80 & $\begin{array}{l}\text { CUC } \\
\text { CUD }\end{array}$ & $\begin{array}{l}97,0 \\
96,4\end{array}$ & $\begin{array}{l}85,8 \\
81,0\end{array}$ & $\begin{array}{l}64,9 \\
44,5\end{array}$ & $\begin{array}{l}46,4 \\
20,8\end{array}$ & $\begin{array}{l}83,6 \\
68,6\end{array}$ & $\begin{array}{l}63,8 \\
46,6\end{array}$ & $\begin{array}{l}45,8 \\
18,5\end{array}$ & $\begin{array}{l}56,5 \\
30,4\end{array}$ & $\begin{array}{l}38,8 \\
14,0\end{array}$ & $\begin{array}{r}24,0 \\
9,3\end{array}$ & 6 \\
\hline 100 & $\begin{array}{l}\text { CUC } \\
\text { CUD }\end{array}$ & $\begin{array}{l}89,3 \\
80,4\end{array}$ & $\begin{array}{l}72,7 \\
65,5\end{array}$ & $\begin{array}{l}53,9 \\
16,9\end{array}$ & $\begin{array}{r}37,4 \\
3,7\end{array}$ & $\begin{array}{l}66,3 \\
41,9\end{array}$ & $\begin{array}{l}47,2 \\
20,7\end{array}$ & $\begin{array}{r}27,3 \\
3,3\end{array}$ & $\begin{array}{r}28,6 \\
5,4\end{array}$ & $\begin{array}{r}12,6 \\
0,5\end{array}$ & $\begin{array}{c}0,0 \\
0,0\end{array}$ & 5 \\
\hline
\end{tabular}

Legenda: Conjuntos de dados (CUC e CUD) com hachuras representam valores combinados considerados excelentes por Mantovani (2001)

Tabela 7. Valores médios obtidos de CUC (\%), CUD (\%) e raio de molhamento (Raio) para a pressão de $300 \mathrm{kPa}$ em função do espaçamento entre aspersores, calculados pelo aplicativo $\mathrm{CATCH}-3 \mathrm{D}$

\begin{tabular}{|c|c|c|c|c|c|c|c|c|c|c|c|c|}
\hline \multirow{2}{*}{$\begin{array}{c}\text { Defletor } \\
(\%)\end{array}$} & \multirow{2}{*}{ CUC e CUD } & \multicolumn{10}{|c|}{ Espaçamento entre aspersores $(\mathrm{m} \times \mathrm{m})$} & \multirow{2}{*}{$\begin{array}{l}\text { Raio } \\
\text { (m) }\end{array}$} \\
\hline & & $6 \times 6$ & $6 \times 8$ & $6 \times 10$ & $6 \times 12$ & $8 \times 8$ & $8 \times 10$ & $8 \times 12$ & $10 \times 10$ & $10 \times 12$ & $12 \times 12$ & \\
\hline 0 & $\begin{array}{l}\text { CUC } \\
\text { CUD }\end{array}$ & $\begin{array}{l}95,4 \\
92,9\end{array}$ & $\begin{array}{l}88,7 \\
81,3\end{array}$ & $\begin{array}{l}95,1 \\
92,1\end{array}$ & $\begin{array}{l}88,9 \\
81,6\end{array}$ & $\begin{array}{l}88,1 \\
76,5\end{array}$ & $\begin{array}{l}89,8 \\
84,5\end{array}$ & $\begin{array}{l}87,6 \\
78,0\end{array}$ & $\begin{array}{l}92,0 \\
88,9\end{array}$ & $\begin{array}{l}84,4 \\
69,0\end{array}$ & $\begin{array}{l}81,3 \\
73,7\end{array}$ & 12 \\
\hline 20 & $\begin{array}{l}\text { CUC } \\
\text { CUD }\end{array}$ & $\begin{array}{l}97,1 \\
95,1\end{array}$ & $\begin{array}{l}91,6 \\
85,0\end{array}$ & $\begin{array}{l}95,6 \\
92,0\end{array}$ & $\begin{array}{l}89,0 \\
81,6\end{array}$ & $\begin{array}{l}88,5 \\
78,0\end{array}$ & $\begin{array}{l}90,5 \\
82,7\end{array}$ & $\begin{array}{l}86,7 \\
77,0\end{array}$ & $\begin{array}{l}93,6 \\
92,6\end{array}$ & $\begin{array}{l}85,5 \\
73,8\end{array}$ & $\begin{array}{l}83,2 \\
77,2\end{array}$ & 12 \\
\hline 50 & $\begin{array}{l}\text { CUC } \\
\text { CUD }\end{array}$ & $\begin{array}{l}97,7 \\
96,3\end{array}$ & $\begin{array}{l}91,2 \\
88,3\end{array}$ & $\begin{array}{l}97,6 \\
96,0\end{array}$ & $\begin{array}{l}93,0 \\
89,2\end{array}$ & $\begin{array}{l}86,8 \\
76,7\end{array}$ & $\begin{array}{l}88,2 \\
85,4\end{array}$ & $\begin{array}{l}87,9 \\
76,7\end{array}$ & $\begin{array}{l}90,3 \\
85,8\end{array}$ & $\begin{array}{l}85,8 \\
79,1\end{array}$ & $\begin{array}{l}88,2 \\
82,9\end{array}$ & 11 \\
\hline 80 & $\begin{array}{l}\text { CUC } \\
\text { CUD }\end{array}$ & $\begin{array}{l}95,8 \\
94,0\end{array}$ & $\begin{array}{l}90,5 \\
87,2\end{array}$ & $\begin{array}{l}72,0 \\
61,9\end{array}$ & $\begin{array}{l}54,6 \\
34,4\end{array}$ & $\begin{array}{l}88,8 \\
82,8\end{array}$ & $\begin{array}{l}72,0 \\
61,0\end{array}$ & $\begin{array}{l}54,6 \\
33,7\end{array}$ & $\begin{array}{l}67,1 \\
47,5\end{array}$ & $\begin{array}{l}50,3 \\
27,7\end{array}$ & $\begin{array}{l}38,1 \\
19,8\end{array}$ & 7 \\
\hline 100 & $\begin{array}{l}\text { CUC } \\
\text { CUD }\end{array}$ & $\begin{array}{l}87,3 \\
80,4\end{array}$ & $\begin{array}{l}72,3 \\
57,3\end{array}$ & $\begin{array}{l}51,1 \\
23,9\end{array}$ & $\begin{array}{r}33,1 \\
9,6\end{array}$ & $\begin{array}{l}70,7 \\
46,5\end{array}$ & $\begin{array}{l}48,5 \\
23,0\end{array}$ & $\begin{array}{r}28,5 \\
8,6\end{array}$ & $\begin{array}{l}37,1 \\
15,8\end{array}$ & $\begin{array}{r}22,4 \\
4,0\end{array}$ & $\begin{array}{l}9,1 \\
0,8\end{array}$ & 6 \\
\hline
\end{tabular}

Legenda: Conjuntos de dados (CUC e CUD) com hachuras representam valores combinados e considerados excelentes, por Mantovani (2001)

$12 \mathrm{~m}$ ) nas pressões de 150, 200,300 e $400 \mathrm{kPa}$, respectivamente, calculados pelo aplicativo computacional CATCH-3D.

$\mathrm{Na}$ Tabela 5, que representa os valores obtidos a partir da pressão $150 \mathrm{kPa}$, os valores de CUC e CUD foram superiores apenas aos preconizados como "Excelente" pela classificação proposta por Mantovani (2001) em 6 e 10\% dos dados, respectivamente; para $200 \mathrm{kPa}$ (Tabela 6) foram encontrados $16 \%$ em ambos os coeficientes; para $300 \mathrm{kPa}$ (Tabela 7), $30 \mathrm{e}$ $32 \%$ e 46 e $52 \%$, respectivamente, para $400 \mathrm{kPa}$ (Tabela 8 ).

Em $23 \%$ dos conjuntos de dados (CUC e CUD) nos espaçamentos estudados, identificados nas Tabelas 5 a 8 por hachuras, ocorreram valores preconizados por Mantovani (2001) como "Excelentes".

Rezende et al. (1998) e Faria et al. (2009) obtiveram, em seus estudos, resultados similares a esses, observando que espaçamentos reduzidos apresentaram melhores valores de CUC. Entretanto, é válido acrescentar que a água aplicada ao solo sofre redistribuição ao se infiltrar (Hart, 1972); além disso e de acordo com Pereira (2003), pequenos espaçamentos aumentam os custos do sistema com tubulações e aspersores e requerem maior mão-de-obra para mudar as linhas de distribuição. 
Tabela 8. Valores médios obtidos de CUC (\%), CUD (\%) e raio de molhamento (Raio) para a pressão de $400 \mathrm{kPa}$ em função do espaçamento entre aspersores, calculados pelo aplicativo CATCH-3D

\begin{tabular}{|c|c|c|c|c|c|c|c|c|c|c|c|c|}
\hline \multirow{2}{*}{$\begin{array}{c}\text { Defletor } \\
(\%)\end{array}$} & \multirow{2}{*}{ CUC e CUD } & \multicolumn{10}{|c|}{ Espaçamento entre aspersores (m x m) } & \multirow{2}{*}{$\begin{array}{l}\text { Raio } \\
\text { (m) }\end{array}$} \\
\hline & & $6 \times 6$ & $6 \times 8$ & $6 \times 10$ & $6 \times 12$ & $8 \times 8$ & $8 \times 10$ & $8 \times 12$ & $10 \times 10$ & $10 \times 12$ & $12 \times 12$ & \\
\hline 0 & $\begin{array}{l}\text { CUC } \\
\text { CUD }\end{array}$ & $\begin{array}{l}96,0 \\
93,6\end{array}$ & $\begin{array}{l}93,6 \\
91,6\end{array}$ & $\begin{array}{l}97,4 \\
95,3\end{array}$ & $\begin{array}{l}90,1 \\
83,2\end{array}$ & $\begin{array}{l}91,9 \\
84,9\end{array}$ & $\begin{array}{l}92,8 \\
90,6\end{array}$ & $\begin{array}{l}88,9 \\
79,1\end{array}$ & $\begin{array}{l}93,3 \\
88,9\end{array}$ & $\begin{array}{l}88,1 \\
77,0\end{array}$ & $\begin{array}{l}86,3 \\
81,9\end{array}$ & 12 \\
\hline 20 & $\begin{array}{l}\text { CUC } \\
\text { CUD }\end{array}$ & $\begin{array}{l}98,6 \\
98,3\end{array}$ & $\begin{array}{l}92,9 \\
89,8\end{array}$ & $\begin{array}{l}97,5 \\
95,9\end{array}$ & $\begin{array}{l}91,4 \\
86,3\end{array}$ & $\begin{array}{l}91,8 \\
84,8\end{array}$ & $\begin{array}{l}93,2 \\
90,0\end{array}$ & $\begin{array}{l}89,9 \\
81,7\end{array}$ & $\begin{array}{l}92,9 \\
89,4\end{array}$ & $\begin{array}{l}87,6 \\
77,3\end{array}$ & $\begin{array}{l}85,4 \\
80,0\end{array}$ & 12 \\
\hline 50 & $\begin{array}{l}\text { CUC } \\
\text { CUD }\end{array}$ & $\begin{array}{l}95,6 \\
93,2\end{array}$ & $\begin{array}{l}92,5 \\
89,4\end{array}$ & $\begin{array}{l}95,0 \\
91,0\end{array}$ & $\begin{array}{l}92,4 \\
88,4\end{array}$ & $\begin{array}{l}91,2 \\
84,3\end{array}$ & $\begin{array}{l}91,8 \\
86,6\end{array}$ & $\begin{array}{l}89,8 \\
79,9\end{array}$ & $\begin{array}{l}90,8 \\
85,1\end{array}$ & $\begin{array}{l}89,0 \\
84,1\end{array}$ & $\begin{array}{l}89,4 \\
85,9\end{array}$ & 11 \\
\hline 80 & $\begin{array}{l}\text { CUC } \\
\text { CUD }\end{array}$ & $\begin{array}{l}95,3 \\
92,6\end{array}$ & $\begin{array}{l}89,7 \\
86,5\end{array}$ & $\begin{array}{l}72,1 \\
63,7\end{array}$ & $\begin{array}{l}56,9 \\
40,0\end{array}$ & $\begin{array}{l}88,6 \\
85,4\end{array}$ & $\begin{array}{l}72,1 \\
64,7\end{array}$ & $\begin{array}{l}57,4 \\
38,6\end{array}$ & $\begin{array}{l}68,5 \\
52,3\end{array}$ & $\begin{array}{l}52,6 \\
32,3\end{array}$ & $\begin{array}{l}42,3 \\
24,2\end{array}$ & 7 \\
\hline 100 & $\begin{array}{l}\text { CUC } \\
\text { CUD }\end{array}$ & $\begin{array}{l}92,5 \\
88,9\end{array}$ & $\begin{array}{l}84,5 \\
80,4\end{array}$ & $\begin{array}{l}65,3 \\
44,7\end{array}$ & $\begin{array}{l}49,0 \\
21,4\end{array}$ & $\begin{array}{l}78,7 \\
65,6\end{array}$ & $\begin{array}{l}64,3 \\
46,9\end{array}$ & $\begin{array}{l}45,2 \\
20,1\end{array}$ & $\begin{array}{l}49,8 \\
29,0\end{array}$ & $\begin{array}{l}34,0 \\
14,8\end{array}$ & $\begin{array}{r}20,0 \\
9,6\end{array}$ & 7 \\
\hline
\end{tabular}

Legenda: Conjuntos de dados (CUC e CUD) com hachuras representam valores combinados e considerados excelentes por Mantovani (2001)

Observa-se redução dos valores de CUC e CUD com o aumento do espaçamento dos aspersores. Holanda Filho et al. (2001) encontraram resultados semelhantes em relação aos valores de CUC com o microaspersor Rondo $\left(50 \mathrm{~L} \mathrm{~h}^{-1}\right)$ da empresa Plastro. Pode ser observado, também, que o aumento da pressão acarretou melhoria para valores de CUC e do CUD entre os espaçamentos estudados, com resultados satisfatórios no espaçamento 10 x $10 \mathrm{~m}$ nas pressões de 300 e $400 \mathrm{kPa}$ com o defletor em até $50 \%$, em conformidade com Pereira (2003), ao citar que o ideal é utilizar espaçamentos médios entre aspersores no campo.

Nas Figuras 5, 6, 7 e 8 são representados, graficamente, os perfis de distribuição de água para todas as posições do defletor

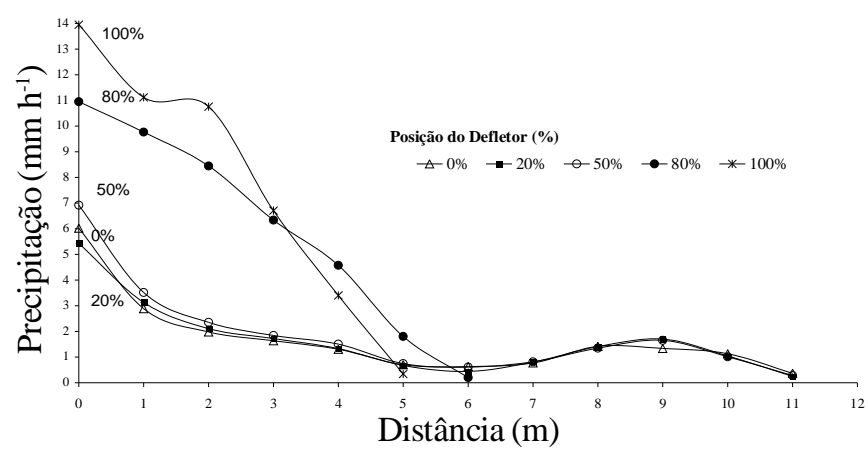

Figura 5. Perfis de distribuição de água do aspersor $\mathrm{N}$ aanDanjain $427 \mathrm{com}$ bocal de 2,8 $\mathrm{mm}$ operando com diferentes posições do defletor de ajuste e pressão de $150 \mathrm{kPa}$

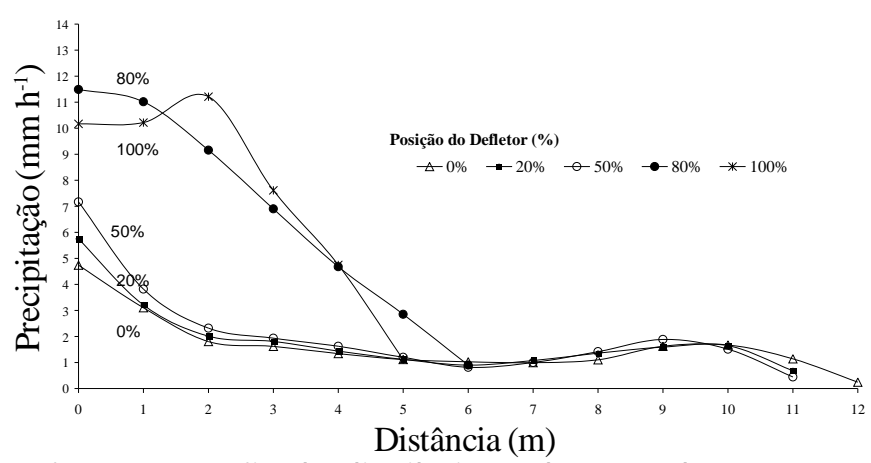

Figura 6. Perfis de distribuição de água do aspersor $\mathrm{N}$ aanDanjain $427 \mathrm{com}$ bocal de 2,8 $\mathrm{mm}$ operando com diferentes posições do defletor de ajuste e pressão de 200 kPa e pressões estudadas. Observou-se que em todas as pressões estudadas as posições de 0,20 e $50 \%$ do defletor de ajuste apresentaram maiores valores de diâmetro de molhamento e perfis de distribuição de água mais uniformes com precipitações máximas variando de 4,7 a 7,0 $\mathrm{mm} \mathrm{h}^{-1}$ junto à haste do aspersor.

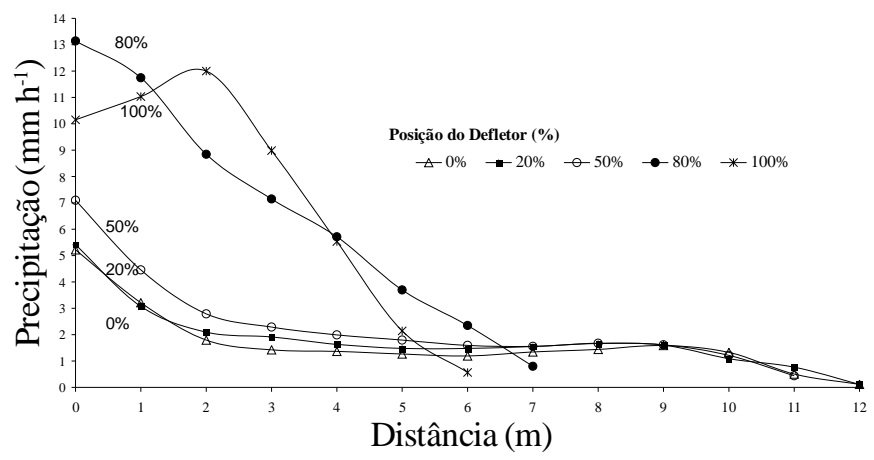

Figura 7. Perfis de distribuição de água do aspersor $\mathrm{N}$ aanDanjain $427 \mathrm{com}$ bocal de $2,8 \mathrm{~mm}$ operando com diferentes posições do defletor de ajuste e pressão de $300 \mathrm{kPa}$

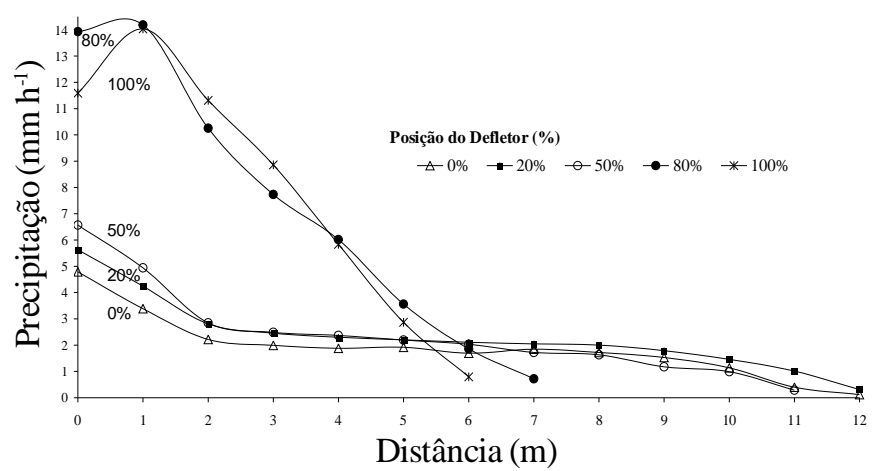

Figura 8. Perfis de distribuição de água do aspersor NaanDanJain 427 com bocal de 2,8 mm operando com diferentes posições do defletor de ajuste e pressão de $400 \mathrm{kPa}$

Pode-se constatar também, nas Figuras 5, 6, 7 e 8 que, predominantemente e sempre que houve variação na posição do defletor de ajuste da posição de $0 \%$ até a posição $100 \%$, ocorreu incremento no volume de água aplicado nas proximidades do aspersor, sobretudo dentro do raio de $2,0 \mathrm{~m}$ a partir do aspersor. 
Em relação ao raio de alcance do jato do aspersor, observase que os valores decresceram à medida em que o comprimento do defletor de ajuste foi aumentado, indicando influência direta do defletor na distribuição de água devido à interceptação e fracionamento do jato d'água que sai através do bocal do aspersor; apesar disto e conforme a ABNT (1999), a intensidade de precipitação em cada coletor deve ser superior a $0,25 \mathrm{~mm} \mathrm{~h}^{-1}$ para se considerar o raio molhado do aspersor, premissa esta não atendida pelo aspersor nas pressões estudadas em relação à informação apresentada no catálogo, pelo fabricante.

O defletor de ajuste mostrou-se como mecanismo interessante, pois permite o comportamento diferenciado do aspersor, possibilitando ampla faixa de sua utilização em situações de interesse prático; entretanto, é importante que o fabricante coloque em seu catálogo informações sobre a distribuição de água do aspersor em função da posição do defletor de ajuste.

\section{ConClusões}

1. As combinações entre as posições do defletor de ajuste e as pressões estudadas influenciam no raio de molhamento do aspersor, na uniformidade de aplicação e no perfil de distribuição de água.

2. O raio de molhamento praticamente não é influenciado até a posição $50 \%$, porém se reduz à metade do alcance máximo com 80 ou $100 \%$ da expansão do defletor, reduzindo a uniformidade de distribuição de água.

3. O aumento da pressão de serviço aumentou a uniformidade de distribuição de água do aspersor.

4. Para alta uniformidade de distribuição de água e maiores raios de molhamento, o aspersor deve trabalhar nas pressões entre 300 e $400 \mathrm{kPa}$, podendo-se utilizar espaçamentos de até $10 \times 10 \mathrm{~m}$.

5. O defletor nas posições 0,20 e $50 \%$ combinadas com os espaçamentos simulados, apresenta as melhores uniformidades de distribuição de água.

\section{LITERATURA CITADA}

ABNT - Associação Brasileira de Normas Técnicas. Equipamentos de irrigação agrícola: Aspersores rotativos. Parte 1: Requisitos para projetos e operação. Projeto 04:015.08-012. Parte 2: Uniformidade de distribuição e métodos de ensaio. Projeto 04:015.08-013. Rio de Janeiro: ABNT, 1999. 22p.

Allen, R. G. Catch-3D sprinkler pattern analysis software: user's manual. Logan: Utah State University, Departament of Biological and Irrigation Engineering, 1992. 14p.

Álvarez, J. F. O.; Martin-Bento, J. M. T.; Varelo, J. A. J.; Pérez, P. C. Uniformity distribution and its economic effects on irrigation management in semiarid zones. Journal of Irrigation and Drainage Engineering, v.130, p.257-268, 2004.

Andrade, E. M.; D’Almeida, D. M. B. A.; Meireles, A. C. M.; Lemos Filho, L. C. de A.; Arruda, F. E. R. Evolução da concentração iônica da solução do solo em áreas irrigadas na Chapada Apodi, CE. Revista Ciência Agronômica, v.35, p.9-16, 2004.
Borges Júnior, J. C. F.: Ferreira, P. A.; Hedden-Dunkhorst, B.; Andrade, C. L. T. Modelo computacional para suporte à decisão em áreas irrigadas: Parte I: Desenvolvimento e análise de sensibilidade. Revista Brasileira de Engenharia Agrícola e Ambiental, v.12, p.3-11, 2008.

Capra, A.; Scicolone, B. Emiterandfiltertest for wastewater reuse bydripirrigation. Agricultural Water Menangement, v.68, p.135-149, 2004.

Christiansen, J. E. Irrigation by sprinkler. Berkeley: California Agricultural Station, 1942. 212p. Bulletin 670

Christofidis, D. Água, irrigação e segurança alimentar. Revista Item, v.77, p.19-21, 2008.

Coelho, E. F.; Coelho Filho, M. A.; Oliveira, S. L. Agricultura irrigada: eficiência de irrigação e de uso de água. Revista Bahia Agrícola, v.7, p.57-60, 2005.

Criddle, W. D.; Davis, S.; Pair, C. H.; Shockeley, D. G. Methods for evaluating irrigation systems. Washington: SCS-USDA, 1956. 24p. Agricultural Handbook, 82.

Faria, L. C.; Colombo, A.; Oliveira, H. F. E. de; Prado, G. Simulação da uniformidade da irrigação de sistemas convencionais de aspersão operando sob diferentes condições de vento. EngenhariaAgrícola, v.29, p.19-27, 2009.

Hart, W. E. Subsurface distribution of nonuniformity applied surface waters. Transactions of ASAE, v.15, p.656-661, 1972.

Hart, W. E.; Peri, G.;Skogerboe, G. V. Irrigation performance: an evaluation. Journal of the Irrigation and Drainage Division. v.105, p.275-288, 1979.

Holanda Filho, R. S. F. de; Porto Filho, F. de Q; Miranda, N. de O.; Medeiros, J. F. de. Caracterização hidráulica do microaspersor Rondo, da Plastro. Revista Brasileira de Engenharia Agrícola e Ambiental, v.5, p.16-21, 2001.

Keller, J.; Karmeli, D. Trickle irrigation design parameters.Transactions of ASAE, v.17, p.678-694, 1974.

Louie, M.; Selker, J. S. Sprinkler head maintenance effects on water application uniformity. Journal of Irrigation and Drainage Engineering, v.126, p.142-148. 2000.

Mantovani, E. C. AVALIA: Programa de avaliação da irrigação por aspersão e localizada. Viçosa: UFV, 2001.

NaanDanJain. Aspersor 427 1/2": Especificações técnicas. <http://www.naandanjain.com.br/produto.aspx?id=97>. 10 Set. 2010.

Oliveira, H. F. E. de; Colombo, A.; Faria, L. C. Modelagem dos efeitos do vento sobre as dimensões do alcance do jato de um canhão hidráulico. Revista Brasileira de Engenharia Agrícola e Ambiental, v.13,p.818-824, 2009.

Paulino, M. A. de O.; Figueiredo, F. P. de; Fernandes, R. C.; Maia, J. T. L. S.; Guilherme, D. de O.; Barbosa, F. S. Avaliação da uniformidade e eficiência de aplicação de água em sistemas de irrigação por aspersão convencional. Revista Brasileira de Agricultura Irrigada, v.3, p.48-54, 2009.

Pereira, G. M. Aspersão Convencional. In: Miranda, J. H.; Pires, R. C. M. Irrigação. Piracicaba: FUNEP, 2003. v.2, 703p.

Prado, G.; Colombo, A. Composição de perfis radiais de distribuição de água de aspersores. Irriga, v. 14, p.41-53, 2009.

Rezende, R.; Frizzone, J. A.; Gonçalves, A. C. A.; Freitas, P. S. L. de. Influência do espaçamento entre aspersores na uniformidade de distribuição de água acima e abaixo da superfície do solo. Revista Brasileira de Engenharia Agrícola eAmbiental, v.2, p.257-261, 1998. 